\title{
Measurement of Epicardial Adipose Tissue (EAT) Thickness in Subclinical Hypothyroid (SH) Patients and To Determine The Relationship Between EAT and Abdominal /Visceral Fat Mass.
}

Dilek Arpaci1, Aysel Gurkan Tocoğlu1, Sabiye Yilmaz2, Sumeyye Korkmaz1, Hasan Ergenc1, Huseyin Gunduz2, Nurgul Keser2, Ali Tamer1 1-Sakarya University, Sakarya Education and Research Hospital, Deparment of Internal Medicine, Sakarya, Turkey

2- Sakarya University, Sakarya Education and Research Hospital, Deparment of Cardiology, Sakarya, Turkey

\section{Background}

Subclinical hypothyroidism (SH) is defined as high thyroid stimulating hormone (TSH) accompanied with normal free thyroxine (FT4). SH has found to be associated with cardiovascular disease (CVD) and increased mortality. EAT is now considered to be a new noninvasive measurement method used for the early detection of CVD susceptibility. EAT has vasocrine and paracrine actions which lead to CVD. We aimed to evaluate measurement of EAT and to detect relationship between EAT and abdominal/visceral fat mass in patients with $\mathrm{SH}$.

\section{Materials/Methods}

This study included 41 patients with SH and 35 healthy controls (matched in age and gender). Demographic data (age, gender, body mass index (BMI), fat mass (FM), abdominal FM, visceral FM, waist circumstance (WC), hip circumstances (HC), systolic and diastolic blood pressure) and laboratory results [fasting plasma glucose (FPG), low density cholesterol (LDL-c); high-density cholesterol (HDLC); triglycerides (TGS); FT4, TSH and anti-TPO] were recorded. EAT was measured with transthoracic echocardiography.

\section{Results}

Fat mass, BMI, $\mathrm{WC}$ and $\mathrm{WC} / \mathrm{HC}$ ratio, visceral and abdominal fat mass were higher in the study group than the control group $(\mathrm{P}<0.05)$. While there were no differences in terms of FPG $(p=0.780)$, there were significant differences regarding LDL-C and TGs between the two groups ( $p=0.002$ and $p=0.026$, respectively). Serum TSH and anti-TPO levels were found higher and FT4 level was lower in the study group than the control group $(\mathrm{p}<0.05)$. Although there were significant differences in terms of BMI, $\mathrm{FM}$, abdominal and visceral $\mathrm{FM}, \mathrm{WC}, \mathrm{HC}, \mathrm{WC} / \mathrm{HC}$ ratio, LDL-C and TG, there were no differences in EAT between the two groups (Table 1). Mean EAT was $4.61 \pm 0.06 \mathrm{~mm}$ in the study group and $4.51 \pm 0.07$ $\mathrm{mm}$ in the control group $(\mathrm{p}=0.532)$. While positive correlation was found between EAT and demographic parameters and serum TG levels $(\mathrm{p}<0.05)$, no correlation was found between EAT and TSH, FT4, LDL-C and anti-TPO level( $\mathrm{p}>0.05$ ) (Table 2).
Table 1: Comparison of demographic and laboratory data between the two groups

\begin{tabular}{|c|c|c|c|}
\hline Feature & $\begin{array}{l}\text { Case } \\
(n=41)\end{array}$ & $\begin{array}{l}\text { Control } \\
(n=35)\end{array}$ & P value \\
\hline Age (year) & $34.07 \pm 6.70$ & $31.82 \pm 5.57$ & 0.121 \\
\hline Gender(F/M) & $39 / 2$ & $35 / 0$ & 0.998 \\
\hline $\operatorname{BMI}\left(\mathbf{k g} / \mathbf{m}^{2}\right)$ & $27.84 \pm 5.26$ & $23.72 \pm 3.01$ & 0.000 \\
\hline Fat mass (\%) & $31.74 \pm 7.58$ & $26.80 \pm 6.42$ & 0.004 \\
\hline Fat mass (kg) & $23.09 \pm 9.89$ & $16.85 \pm 5.99$ & 0.002 \\
\hline $\begin{array}{l}\text { Waist } \\
\text { Circumstances } \\
\text { (WC-cm) }\end{array}$ & $93.68 \pm 12.09$ & $87.94 \pm 8.38$ & 0.038 \\
\hline $\begin{array}{l}\text { (Hip circumstances } \\
\text { (HC-cm) }\end{array}$ & $103.86 \pm 10.37$ & $100.71 \pm 6.73$ & 0.152 \\
\hline $\mathrm{WC} / \mathrm{HC}$ & $0.95 \pm 0.07$ & $0.90 \pm 0.005$ & 0.007 \\
\hline Visceral fat mass & $9.53 \pm 4.03$ & $7.10 \pm 2.40$ & 0.005 \\
\hline $\begin{array}{l}\text { Abdominal fat } \\
\text { mass }\end{array}$ & $40.24 \pm 6.57$ & $35.33 \pm 6.32$ & 0.004 \\
\hline $\begin{array}{l}\text { Systolic } \\
\text { BP(mmHg) }\end{array}$ & $120.10 \pm 7.24$ & $118.97 \pm 7.36$ & 0.471 \\
\hline $\begin{array}{l}\text { Diastolic } \\
\text { BP(mmHg) }\end{array}$ & $62.23 \pm 5.80$ & $60.70 \pm 3.89$ & 0.065 \\
\hline FBG(mg/dl) & $90.68 \pm 6.30$ & $89.12 \pm 7.68$ & 0.780 \\
\hline TSH $(\mu I \mathrm{U} / \mathrm{mL})$ & $14.55 \pm 6.50$ & $1.54 \pm 0.82$ & 0.000 \\
\hline FT4 (pmol/L) & $11.60 \pm 2.68$ & $14.15 \pm 1.70$ & 0.000 \\
\hline $\begin{array}{l}\text { Anti-TPO } \\
\text { (IU/mL) }\end{array}$ & $313.65 \pm 358.69$ & $2.42 \pm 1.16$ & 0.000 \\
\hline LDL (mg/dl) & $125.85 \pm 31.73$ & $104.39 \pm 25.50$ & 0.002 \\
\hline TG(mg/dl) & $105.90 \pm 50.08$ & $83.75 \pm 27.17$ & 0.026 \\
\hline HDL ( mg/dl) & $55.21 \pm 15.40$ & $57.32 \pm 11.79$ & 0.495 \\
\hline EAT(mm) & $4.61 \pm 0.06$ & $4.51 \pm 0.07$ & 0.532 \\
\hline
\end{tabular}

\begin{tabular}{|l|ll|} 
& \multicolumn{2}{|c|}{ Total $(\mathrm{n}=76)$} \\
& r value & P value \\
\hline BMI(kg/m²) & 0.402 & 0.000 \\
\hline Fat Mass $(\mathrm{kg})$ & 0.408 & 0.000 \\
\hline WC(cm) & 0.400 & 0.000 \\
\hline HC(cm) & 0.340 & 0.001 \\
\hline Visseral Fat Mass $(\mathrm{kg})$ & 0.321 & 0.002 \\
\hline Abdominal Fat Mass(Kg) & 0.345 & 0.001 \\
\hline TSH (uII/mL) & -0.094 & 0.328 \\
\hline FT4(pmo/L) & 0.025 & 0.794 \\
\hline Anti-TPO(IIU/mL) & 0.202 & 0.088 \\
\hline LDL-C(mg/dl) & 0.028 & 0.779 \\
\hline TGss(mg/dl) & 0.270 & 0.006 \\
\hline
\end{tabular}

\section{Conclusion}

Despite EAT has been playing an important role in predicting subclinical atherosclerosis in $\mathrm{SH}$, this study could not support this. In our opinion, more studies with more number of patients are needed to claim that. 